\title{
Efficacy and Safety of Pyronaridine-Artesunate for the Treatment of Uncomplicated Plasmodium falciparum and Plasmodium vivax Malaria in Myanmar
}

\author{
Kay Thwe Han, ${ }^{1} \dagger$ Khin Lin, ${ }^{2} \dagger$ Zay Yar Han, ${ }^{1}$ Moe Kyaw Myint, ${ }^{2}$ Kyin Hla Aye, ${ }^{1}$ Aung Thi, ${ }^{3}$ Badri Thapa, ${ }^{4}$ Maria Dorina Bustos, ${ }^{5}$ \\ Isabelle Borghini-Fuhrer, ${ }^{6}$ Pascal Ringwald, ${ }^{7}$ and Stephan Duparc ${ }^{6 *}$ \\ ${ }^{1}$ Department of Medical Research, Ministry of Health and Sports, Yangon, Myanmar; ${ }^{2}$ Department of Medical Research (Pyin Oo Lwin Branch), \\ Ministry of Health and Sports, Pyin Oo Lwin Township, Myanmar; ${ }^{3}$ National Malaria Control Programme, Department of Public Health, Ministry of \\ Health and Sports, Nay Pyi Taw, Myanmar; ${ }^{4}$ World Health Organization, Yangon, Myanmar; ${ }^{5}$ World Health Organization, Bangkok, Thailand; \\ ${ }^{6}$ Medicines for Malaria Venture, Geneva, Switzerland; ${ }^{7}$ World Health Organization, Geneva, Switzerland
}

\begin{abstract}
Four single-arm, prospective, clinical studies of pyronaridine-artesunate efficacy in uncomplicated Plasmodium falciparum or Plasmodium vivax malaria were conducted in Myanmar between 2017 and 2019. Eligible subjects were aged at least 6 years, with microscopically confirmed $P$. falciparum $(n=196)$ or $P$. vivax mono-infection $(n=206)$. Patients received pyronaridine-artesunate once daily for 3 days with follow-up until day 42 for $P$. falciparum or day 28 for $P$. vivax. For the primary efficacy analysis, adequate clinical and parasitological response (ACPR) in the per-protocol population at day 42 for $P$. falciparum malaria was 100\% (88/88; 95\% Cl: 95.9, 100) in northern Myanmar (Kachin State and northern Shan State), and 100\% (101/101; 95\% Cl: 96.4, 100) in southern Myanmar (Tanintharyi Region and Kayin State). Plasmodium falciparum day-3 parasite clearance was observed for $96.9 \%(190 / 196)$ of patients. Mutations in the P. falciparum Kelch propeller domain (K13) were detected in 39.0\% (69/177) of isolates: F446I (14.7\% [26/177]), R561H (13.0\% [23/177]), C580Y (10.2\% [18/177]), and P574L (1.1\% [2/177]). For P. vivax, the day-28 ACPR was 100\% (104/104; 95\% Cl: $96.5,100)$ in northern Myanmar and 100\% $(97 / 97 ; 95 \% \mathrm{Cl}: 96.3,100)$ in southern Myanmar. Across both P. vivax studies, $100 \%(206 / 206)$ of patients had day-3 parasite clearance. There were no adverse events. Pyronaridineartesunate had excellent efficacy in Myanmar against $P$. falciparum and $P$. vivax and was well tolerated. This study supports the inclusion of pyronaridine-artesunate in national malaria treatment guidelines for Myanmar.
\end{abstract}

\section{INTRODUCTION}

The effective treatment of malaria is a priority health target in Myanmar, and the country has committed to eliminating falciparum malaria by 2025 and all kinds of malaria by 2030 . Case management is used in areas targeted for elimination, and rapid diagnostic testing and treatment with artemisinin-based combination therapy (ACT) are provided free of charge by public and private clinics. The current first-line agent for Plasmodium falciparum malaria is artemether-lumefantrine, followed by single-dose primaquine $(0.75$ $\mathrm{mg} / \mathrm{kg}$ ). Dihydroartemisinin-piperaquine or artesunate-mefloquine are available as altemative ACT regimens. Plasmodium vivax malaria is treated with chloroquine complemented by radical $\mathrm{Cu}-$ rative treatment with primaquine ( $0.25 \mathrm{mg} / \mathrm{kg} /$ day for 14 days).

Myanmar is one of the six countries with known artemisininresistant $P$. falciparum. ${ }^{1}$ The artemisinin resistance phenotype is defined as an increase in day-3 parasite positivity following ACT treatment, that is, a delay in parasite clearance. ${ }^{1,2}$ Clinical efficacy is not affected unless resistance also develops to the partner drug, but this is more likely to emerge and to spread once the antimalarial activity of the artemisinin component is compromised. Unfortunately, multidrug-resistant $P$. falciparum is a serious problem across the Greater Mekong Subregion (GMS). ${ }^{3,4}$ Thus, potential alternative therapeutic options for Myanmar require evaluation in case current first-line treatments for falciparum malaria become ineffective, as observed in Cambodia. ${ }^{3,4}$

The ACT pyronaridine-artesunate has shown good efficacy for uncomplicated falciparum and vivax malaria in large-scale clinical trials conducted in Asia and Africa. ${ }^{5-12}$ Within the GMS, pyronaridine-artesunate PCR-corrected day-42 adequate clinical

\footnotetext{
* Address correspondence to Stephan Duparc, Medicines for Malaria Venture, P. O. Box 1826 20, Route de Pré-Bois, Geneva 1215, Switzerland. E-mail: duparcs@mmv.org
}

$\dagger$ These authors contributed equally to this work. and parasitological response (ACPR) rates against falciparum malaria in western Cambodia were $87.9 \%$ (95\% Cl: 80.6, 93.2) in 2014-2015, ${ }^{13}$ but more recently $>98 \%$ efficacy was noted for this region (2018) ${ }^{14}$ and efficacy was $>96 \%$ in eastern Cambodia (1997), ${ }^{15}$ and $>96 \%$ in Vietnam (2017-2018). ${ }^{16}$ Thus, pyronaridine-artesunate can remain a valuable treatment option for uncomplicated falciparum malaria in regions where the utility of other antimalarial drugs has been compromised by drug resistance.

Intensive malaria control and treatment efforts in Myanmar have reduced the prevalence of $P$. falciparum. ${ }^{17}$ However, $P$. vivax remains refractory to current interventions and has become the dominant parasite in some areas. ${ }^{18,19}$ Pyronaridine-artesunate is the first ACT specifically registered for $P$. vivax malaria, with demonstrated high clinical efficacy across Southeast Asia. ${ }^{12,14}$ Chloroquine resistance has been reported in Myanmar, ${ }^{20-23}$ and to plan effective $P$. vivax elimination programs, the efficacy of alternative therapies requires confirmation.

This study assessed the efficacy of pyronaridine-artesunate for the treatment of uncomplicated $P$. falciparum and $P$. vivax malaria in southern and northern Myanmar to support a review of the national malaria treatment policy and to inform the design of malaria elimination programs in the context of artemisinin resistance.

\section{MATERIALS AND METHODS}

Study design and ethics. Four single-arm, prospective, antimalarial treatment efficacy studies were conducted between July and December 2017 in two townships in northern Myanmar (Kachin State and northern Shan State) and between August 2017 and November 2019 in five townships in southern Myanmar (Tanintharyi Region and Kayin State) (Table 1, Figure 1).

The studies complied with the Declaration of Helsinki, Good Clinical Practice guidelines, and all relevant local and 
international laws. The protocol was reviewed and approved by the Ethics Review Committee, Department of Medical Research, Ministry of Health \& Sport, Myanmar, and the WHO Research Ethics Review Committee. All adult participants and parents or guardians of participants aged $<18$ years provided written informed consent; in addition, assent was required from children aged $\geq 12$ years. The trials were registered on the Australian New Zealand Clinical Trials Registry (Table 1). The standard WHO therapeutic efficacy test protocol was used for all four studies, and all were reported using the same methods and analysis. ${ }^{24}$

Participants. Across all four studies, eligible patients had to be at least 6 years of age, have microscopically confirmed uncomplicated malaria with fever or history of fever, be able to swallow oral medication, and be willing to comply with the study protocol. Patients had to have microscopically confirmed mono-infection with $P$. falciparum $(500-100,000 / \mu \mathrm{L}$ asexual parasites) or microscopically confirmed $P$. vivax mono-infection (parasitemia of $250-100,000 / \mu \mathrm{L}$ asexual parasites) (Table 1).

General exclusion criteria were signs and symptoms of severe falciparum malaria, ${ }^{25}$ body weight $<20 \mathrm{~kg}$, mixed Plasmodium infection, severe malnutrition, the presence of febrile conditions other than malaria or other underlying chronic or severe diseases, regular medication which might interfere with antimalarial pharmacokinetics, a history of hypersensitivity reaction, or contraindications to the study medicine. All female participants aged 12-17 years were excluded because pregnancy testing before enrollment was not feasible owing to cultural conditions. Otherwise, women of child-bearing age were excluded if they had a positive pregnancy test, were breastfeeding, or were unwilling to use contraception. In addition, if patients had $P$. vivax infection, they were excluded if their hemoglobin was $<8 \mathrm{~g} / \mathrm{dL}$ (using HemoCue ${ }^{\circledR}$, Ängelholm, Sweden).

Treatment. Pyronaridine-artesunate tablets (180:60 mg pyronaridine-artesunate; Pyramax ${ }^{\circledR}$, Shin Poong Pharmaceutical Co., Ansan, Republic of Korea) were given orally once daily for 3 days (days 0,1 , and 2 ). Dosing was according to the body weight; 20 to $<24 \mathrm{~kg}$, one tablet per day, 24 to $<45 \mathrm{~kg}$, two tablets per day, 45 to $<65 \mathrm{~kg}$, three tablets per day, and $\geq 65 \mathrm{~kg}$, four tablets per day. Patients were treated as outpatients, but all doses were supervised and patients were observed for 30 minutes after dosing. In case of vomiting, patients were re-dosed and if the patient vomited again, they were withdrawn and administered rescue medication. Patients with $P$. falciparum malaria also received single-dose primaquine $(0.75 \mathrm{mg} / \mathrm{kg})$. In the case of $P$. vivax malaria, after testing for glucose-6-phosphate dehydrogenase (G6PD) deficiency using Carestart ${ }^{\mathrm{TM}}$ rapid diagnostic test (AccessBio, Somerset, $\mathrm{NJ}$ ), primaquine was administered according to national treatment guidelines, that is, $0.25 \mathrm{mg} / \mathrm{kg} /$ day for 14 days if the patient was G6PD normal or $0.75 \mathrm{mg} / \mathrm{kg} /$ day once weekly for 8 weeks if they were G6PD deficient.

Procedures. Patients were treated for 3 days (days 0,1 , and 2), with follow-up on days $3,7,14,21,28,35$, and 42 for patients with $P$. falciparum and up to day 28 for those with $P$. vivax. The medical history, including prior and concomitant medication, and demographic information were collected at baseline. Physical examination and clinical assessment were conducted on day 0 before dosing, on days 1, 2, and 3, and at all follow-up visits. Giemsa-stained thick and thin blood films for parasite identification and counts were examined on day 0 and thick blood films were examined on days 1,2, 3, and at all follow-up visits or any day the patient returned. Asexual

TABLE 1

Overview of four surveillance studies evaluating pyronaridine-artesunate therapeutic efficacy conducted in Myanmar during 2017-2019 and patient baseline characteristics

\begin{tabular}{|c|c|c|c|c|}
\hline \multirow[b]{2}{*}{ Description/characteristic } & \multicolumn{2}{|c|}{ P. falciparum } & \multicolumn{2}{|c|}{ P. vivax } \\
\hline & Northern Myanmar & Southern Myanmar & Northern Myanmar & Southern Myanmar \\
\hline Study registration* & ACTRN12618001952235 & ACTRN12618001620213 & ACTRN12618001952235 & ACTRN12618001621202 \\
\hline Study sites & $\begin{array}{l}\text { Kachin State } \\
\text { (Myit Kyi Nar) } \\
\text { Northern Shan State } \\
\text { (Kyauk Mee) }\end{array}$ & $\begin{array}{l}\text { Tanintharyi Region } \\
\text { (Kawthaung and Mawhtaung) } \\
\text { Kayin State } \\
\text { (Myawaddy and } \\
\text { Kyainseikkyi) }\end{array}$ & $\begin{array}{l}\text { Kachin State } \\
\text { (Myit Kyi Nar) } \\
\text { Northern Shan State } \\
\text { (Kyauk Mee) }\end{array}$ & $\begin{array}{l}\text { Tanintharyi Region } \\
\text { (Kawthaung and Bokpyin) } \\
\text { Kayin State } \\
\text { (Myawaddy and } \\
\text { Kyainseikkyi) }\end{array}$ \\
\hline Dates conducted & $\begin{array}{l}\text { July } 1,2017 \text { to December } \\
25,2017\end{array}$ & $\begin{array}{l}\text { August } 9,2017 \text { to November } \\
3,2018\end{array}$ & $\begin{array}{l}\text { July } 1,2017 \text { to September } \\
30,2017\end{array}$ & $\begin{array}{l}\text { May 2, } 2018 \text { to November } \\
2,2019\end{array}$ \\
\hline Treatment & $\begin{array}{l}\text { Pyronaridine-artesunate } \\
\text { once daily for } 3 \text { days plus } \\
\text { single-dose primaquine } \\
(0.75 \mathrm{mg} / \mathrm{kg})\end{array}$ & $\begin{array}{l}\text { Pyronaridine-artesunate } \\
\text { once daily for } 3 \text { days plus } \\
\text { single-dose primaquine } \\
(0.75 \mathrm{mg} / \mathrm{kg})\end{array}$ & $\begin{array}{l}\text { Pyronaridine-artesunate } \\
\text { once daily for } 3 \text { days plus } \\
\text { primaquine }(0.25 \mathrm{mg} / \mathrm{kg} / \\
\text { day for } 14 \text { days })\end{array}$ & $\begin{array}{l}\text { Pyronaridine-artesunate } \\
\text { once daily for } 3 \text { days plus } \\
\text { primaquine }(0.25 \mathrm{mg} / \mathrm{kg} / \\
\text { day for } 14 \text { days })\end{array}$ \\
\hline Number of patients & 90 & 106 & 104 & 102 \\
\hline Male:female & $58: 32$ & $70: 36$ & 69:35 & $65: 37$ \\
\hline $\begin{array}{l}\text { Mean age (years) } \\
\text { (SD) (range) }\end{array}$ & $30.1(12.4)(9-61)$ & $30.3(14.5)(6-59)$ & $33.2(11.2)(10-60)$ & $21.0(13.9)(6-58)$ \\
\hline $\begin{array}{l}\text { Age } \geq 16 \text { years } \\
n(\%)\end{array}$ & $78(86.7)$ & $88(83.0)$ & $92(88.5)$ & 55 (53.9) \\
\hline $\begin{array}{l}\text { Age } 5-15 \text { years, } \\
n(\%)\end{array}$ & $12(13.3)$ & $18(17.0)$ & $12(11.5)$ & $47(46.1)$ \\
\hline $\begin{array}{l}\text { Mean weight (kg) } \\
\text { (SD) (range) }\end{array}$ & $50.2(10.7)(22-64)$ & 49.1 (10.5) (20-65) & $47.4(7.4)(27-60)$ & $61.8(9.9)(44-76)$ \\
\hline $\begin{array}{l}\text { Mean geometric } \\
\text { parasitemia } \\
\text { (range) }\end{array}$ & $8,014(510-37,200)$ & $8,051(989-92,472)$ & $2,481(750-9,854)$ & $1868(266-18,466)$ \\
\hline
\end{tabular}




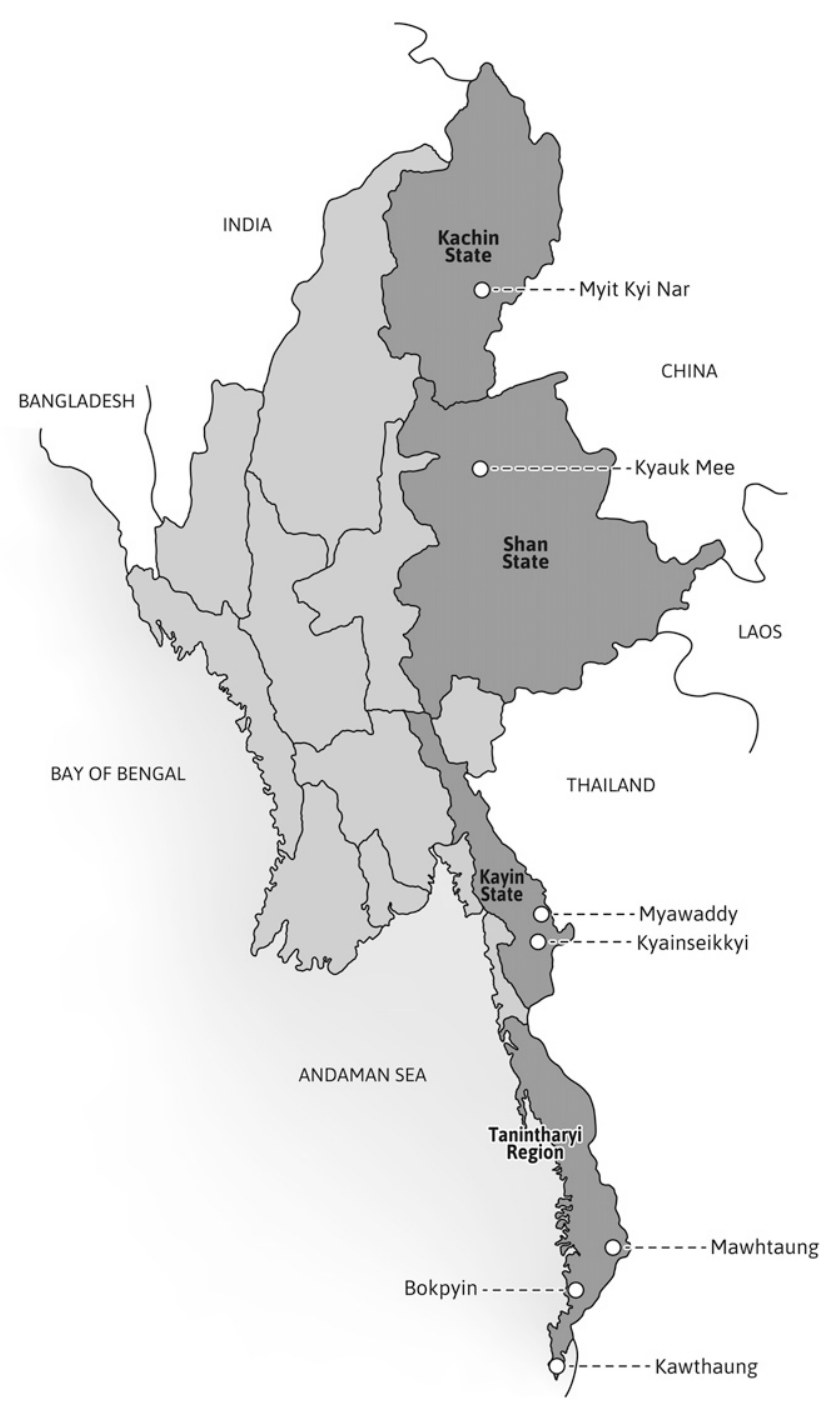

Figure 1. Study sites.

parasites were enumerated using standard methods. ${ }^{26}$ The slides were read by two qualified microscopists and parasite densities calculated as the average of the two counts. Discordant results (differences of $>50 \%$ ) were examined by a third independent microscopist and parasite density calculated as the average of the two closest counts. In the case of $P$. falciparum recurrence, polymerase chain reaction genotyping (PCR) was used to distinguish between recrudescence and reinfection by comparing $P$. falciparum genes $m s p 1$, $m s p 2$, and g/urp in baseline samples versus those obtained at failure, using published methods. ${ }^{27}$ Throughout the follow-up, patients were routinely asked about old symptoms and new symptoms emerging since the previous visit. The nature and incidence of adverse events and serious adverse events were recorded throughout the study.

Molecular surveillance methods. Blood drops were collected on filter paper for assessment of molecular markers of artemisinin resistance in the Kelch propeller domain (K13). Studies were conducted at the laboratory of the Department of Medical Research, Yangon, using published methods. ${ }^{28}$ Briefly, nested PCR was used to amplify $P$. falciparum $K 13$ genes (codon 432-703) from extracted DNA samples, and purified PCR products were sequenced using a $3500 \times \mathrm{L}$ genetic analyzer (ABI Applied Biosystems, Foster City, CA). Sequence analysis of the K13 gene was performed using Sequencher ${ }^{\circledR}$ software (version 5.4, Gene Codes Corporation, Ann Arbor, MI) and BioEdit sequence alignment editor software (version 7.2.5, Informer Technologies, Inc., Roseau Valley, Dominica). The $K 13$ propeller gene sequence for $P$. falciparum 3D7 was retrieved from GenBank (ID; AL844509.2) and used as a reference strain. Fifteen percent of K13 mutant samples and $5 \%$ of wild-type samples were randomly selected and sent to the Pasteur Institute for external quality control; the results were found to be $100 \%$ concordant.

Outcomes. Efficacy outcomes were based on an assessment of the parasitological and clinical outcome of antimalarial treatment according the WHO guidelines. ${ }^{26}$ Patients were classified as having early treatment failure, late clinical failure, late parasitological failure, or ACPR, defined as absence of parasites without previous treatment failure. Adequate clinical and parasitological response was determined at day 42 for $P$. falciparum and day 28 for $P$. vivax. Safety evaluations were the incidence of any adverse event occurring during the study period.

Statistical methods. Efficacy was evaluated using two methods, by calculating the proportion of patients with ACPR in a per-protocol analysis and by Kaplan-Meier estimation of the cumulative incidence of ACPR over the study period. Patients lost to follow-up or withdrawn from the study were excluded from the per-protocol analysis or censored in the Kaplan-Meier analysis at the last available visit. In the PCRadjusted analysis, patients were excluded or censored if the PCR results were unclassifiable or if the results of PCR indicated that the failure was due to reinfection.

For each study, the estimated treatment failure rate for pyronaridine-artesunate was $<5 \%$. For a confidence level of $95 \%$ and a precision around the estimate of $5 \%$, a minimum of 73 patients was required. Allowing for $20 \%$ withdrawals, patients lost to follow-up, and reinfection, the recruitment target was 88 patients per study.

\section{RESULTS}

Patients. In northern Myanmar, 90 patients were treated for P. falciparum malaria, with two lost to follow-up and 104 for $P$. vivax with none lost to follow-up. In southern Myanmar, 106 patients were treated for $P$. falciparum malaria, five of which were lost to follow-up, and 102 patients were treated for $P$. vivax malaria, five of which were lost to follow-up. Patient baseline characteristics are shown in Table 1. The majority of patients with P. falciparum were male $(65.3 \%[128 / 196])$ and older than 16 years $(84.7 \%[166 / 196])$. For $P$. vivax, most patients were male (65.0\% [134/206]) and $71.4 \%(147 / 206)$ were older than 16 years.

Efficacy. For patients with $P$. falciparum in northern Myanmar, day-42 ACPR with pyronaridine-artesunate was $100 \%$ (88/88; $95 \%$ Cl: 95.9, 100) in the per-protocol analysis and 100\% in the Kaplan-Meier analysis (Table 2). In southern Myanmar, P. falciparum day-42 ACPR was 100\% (101/101; 95\% Cl: 96.4, 100 ) in the per-protocol analysis and $100 \%$ using Kaplan-Meier analysis (Table 2). As there were no cases of recurrence, PCR adjustment was unnecessary and PCR-adjusted ACPR was the same (100\% in both regions). Across all enrolled patients from northern Myanmar $(n=90)$, the day-3 parasite positivity rate was $6.7 \%(6 / 90 ; 95 \% \mathrm{Cl}: 2.5,13.9)$. However, all of the patients with parasites at day 3 were from Kachin State, giving a day-3 parasite positivity rate of $13.3 \%(6 / 45 ; 95 \% \mathrm{Cl}: 5.1,26.8)$ for this 
TABLE 2

Pyronaridine-artesunate efficacy against $P$. falciparum and $P$. vivax in Myanmar, per-protocol analysis

\begin{tabular}{|c|c|c|c|c|}
\hline \multirow[b]{2}{*}{ Population and outcome } & \multicolumn{2}{|c|}{ P. falciparum } & \multicolumn{2}{|c|}{ P. vivax } \\
\hline & Northern Myanmar & Southern Myanmar & Northern Myanmar & Southern Myanmar \\
\hline Patients recruited $(n)$ & 90 & 106 & 104 & 102 \\
\hline Lost to follow-up (n) & 2 & 5 & 0 & 5 \\
\hline Per-protocol population (n) & 88 & 101 & 104 & 97 \\
\hline $\begin{array}{l}\text { Adequate clinical and parasitological } \\
\text { response }(\%)(n / N)(95 \% \mathrm{Cl})\end{array}$ & $100(88 / 88)(95.9,100)$ & $100 \%(101 / 101)(96.4,100)$ & $100(104 / 104)(96.5,100)$ & $100(97 / 97)(96.3,100)$ \\
\hline
\end{tabular}

region. All of the 106 enrolled patients from southern Myanmar had parasite clearance at day 3.

For $P$. vivax, pyronaridine-artesunate day-28 ACPR in the per-protocol analysis was 100\% (104/104; 95\% Cl: 96.5, 100) in northern Myanmar and 100\% (97/97; 95\% Cl: $96.3,100)$ in southern Myanmar. In both regions, ACPR was $100 \%$ when estimated using Kaplan-Meier analysis. All 206 enrolled patients across both studies had parasite clearance by day 3 .

Artemisinin resistance molecular surveillance. Plasmodium falciparum $K 13$ sequences were obtained from $92.2 \%$ (83/90) of isolates collected in 2017 in northern Myanmar (Figure 2). The proportion of isolates with $K 13$ mutations was 41.0\% (34/83); 33.3\% (13/39) in Kachin State and 47.7\% (21/ $44)$ in northern Shan State. In both states, the most common mutant was $K 13(\mathrm{R} 561 \mathrm{H})$, with an overall prevalence of $27.7 \%$ (23/83). The C580Y mutant was only detected in northern Shan State (11.4\% [5/44]).

For 2017/2018 data from southern Myanmar, K13 sequences were available for $88.7 \%$ (94/106) of $P$. falciparum isolates (Figure 2). Overall, the $K 13$ mutation rate was $37.2 \%$ (35/94); 30.4\% (14/46) in Tanintharyi Region and 43.8\% (21/ 48) in Kayin State. K13(C580Y) was the most common mutant in Tanintharyi Region, whereas F446I was the dominant mutant in Kayin State (Figure 2).
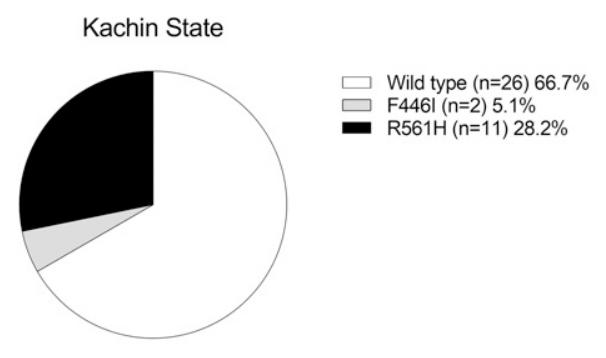

Total $=39$ Tanintharyi Region
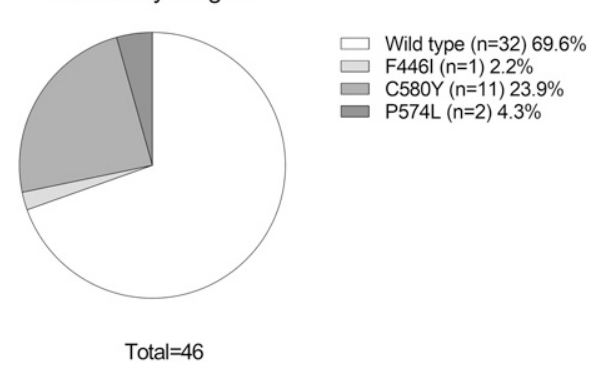

As pyronaridine-artesunate clinical efficacy was $100 \%$, no correlation could be made between $K 13$ mutations and efficacy. Parasitemia at day 3 was only detected in six patients from Kachin State; isolates from two patients had the $K 13(\mathrm{R} 561 \mathrm{H})$ mutation, but the other four isolates were $K 13$ wild type. Thus, there was no relationship between $K 13 \mathrm{mu}-$ tations and day-3 parasite positivity rate.

Safety. There were no adverse events of any cause recorded following treatment with pyronaridine-artesunate in either $P$. falciparum- or $P$. vivax-infected patients.

\section{DISCUSSION}

Malaria elimination in Myanmar is critically dependent on the continued efficacy of ACTs against $P$. falciparum. However, artemisinin-resistant $P$. falciparum is present in the region, and there is the potential for the emergence of partner drug resistance. ${ }^{29,30}$ Thus, the situation is fragile, and the impressive progress that Myanmar has made in reducing malaria prevalence and mortality over the last decade could be easily reversed should multidrug-resistant $P$. falciparum become established. Once a parasite emerges with a clear survival advantage under intense selective pressure from a narrow range of drug therapies, a selective sweep can cause a rapid loss of efficacy. This was seen
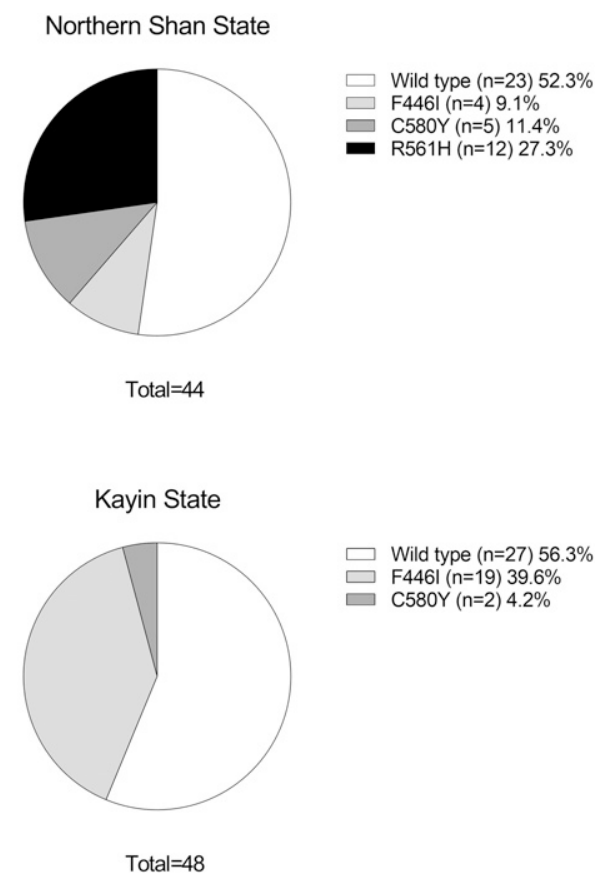

FIGURE 2. Prevalence of Plasmodium falciparum K13 mutations in isolates collected in 2017 from northern Myanmar (Kachin State and northern Shan State) and in 2017/2018 from southern Myanmar (Tanintharyi Region and Kayin State). 
for $P$. falciparum in Cambodia with a dominant artemisinin/ piperaquine multidrug-resistant haplotype emerging within months of dihydroartemisinin-piperaquine being introduced as first-line therapy. ${ }^{3}$ The most recent data from the GMS indicate that pyronaridine-artesunate retains good efficacy in Cambodia and Vietnam against multidrug-resistant $P$. falciparum, ${ }^{14-16}$ and the current study confirms excellent efficacy in Myanmar. These findings are reassuring, potentially allowing the diversification of antimalarial therapy, and suggesting an alternative option should resistance undermine the efficacy of currently recommended antimalarial drugs.

Molecular surveillance of $P$. falciparum $K 13$ mutations suggests a genetically independent artemisinin-resistant parasite population in Thailand-Myanmar-China region versus CambodiaLaos-Vietnam. ${ }^{1,2,31}$ An extensive geospatial mapping study reported the proportion of $K 13$ mutants as $47.6 \%$ for Kayin State, $37.1 \%$ for Kachin State, and $66.7 \%$ for Shan State. ${ }^{32}$ This is broadly consistent with our findings (43.8\%, 33.3\%, and $47.7 \%$, respectively). In the current study, four different $K 13$ mutants were found: F446I, C580Y, and P574L in southern Myanmar and F446I, C580Y, and R561 H in northern Myanmar. All of these are validated artemisinin resistance markers. However, in the current study, the majority of patients were aparasitemic by day 3 , and delayed parasite clearance was associated with $\mathrm{R} 561 \mathrm{H}$ in two cases and wild-type $K 13$ in four cases. These findings are consistent with 2014-2015 data from Rakhine, Shan, and Kachin states, where artemether-lumefantrine and dihydroartemisinin-piperaquine retained high efficacy (>96\% and $100 \%$, respectively), despite the presence of artemisinin-resistant parasites in Shan and Kachin states. ${ }^{33}$ As seen in the current study, the day-3 parasite positivity rate was low following artemether-lumefantrine (0-3.6\%), and all parasites were cleared with dihydroartemisinin-piperaquine. ${ }^{33}$

These studies indicate that in Myanmar, the circulating $K 13$ mutations have limited impact on parasite clearance at present. This is in contrast to Cambodia-Laos-Vietnam, where a multidrugresistant haplotype conferring high-level resistance to artemisinin and piperaquine has reached near fixation in some regions. ${ }^{1,3,14-16}$ Correspondingly, day-3 parasite positivity rates are relatively high, for example, 35.6-46.7\% in eastern regions of Cambodia and 28.6-41.7\% in the west. ${ }^{14,15}$ Moreover, high clinical failure rates are observed for dihydroartemisinin-piperaquine. ${ }^{4} \mathrm{~A}$ limitation of this study was that molecular markers of $P$. falciparum resistance to piperaquine and mefloquine were not investigated. However, there is currently no clinical evidence of drug failure for ACT regimens containing these antimalarial drugs in Myanmar. ${ }^{34}$

The antimalarial resistance situation in Myanmar requires continued close monitoring. However, maintaining effective surveillance in a low-transmission setting is challenging. Finding eligible patients with $P$. falciparum malaria in southern Myanmar was difficult, requiring two transmission seasons to achieve target recruitment. Falciparum malaria prevalence was low in the study areas, with only $1.6 \%(119 / 7,457)$ of patients screened having microscopically confirmed falciparum malaria. Social factors also hampered recruitment, for example, migrant workers in the Myanmar-Thai border area were fearful and reluctant to engage, and a clinic set up by a nongovernmental organization also treated malaria patients in the region.

As the prevalence of $P$. falciparum has declined, $P$. vivax has emerged as a significant barrier to malaria elimination in Myanmar. ${ }^{19,35}$ There is also evidence of $P$. vivax chloroquine resistance in Myanmar, ${ }^{20-23}$ including high-level resistant strains circulating in northeast Myanmar. ${ }^{22}$ Although the prevalence of these strains is thought to be low, the success of elimination efforts based on chloroquine/primaquine could be jeopardized and intensification of $P$. vivax antimalarial treatment may further select and propagate resistant strains. Pyronaridine-artesunate had $100 \%$ efficacy in the current study against $P$. vivax. This is consistent with data from Cambodia, Thailand, India, and Indonesia showing that pyronaridine-artesunate was non-inferior to chloroquine, but with more rapid parasite and fever clearance. ${ }^{12}$ Thus, pyronaridine-artesunate presents a clinically validated alternative to chloroquine for the treatment of $P$. vivax malaria and is the only ACT to receive approval for this indication by a stringent regulatory authority (European Medicines Evaluation Agency). ${ }^{12}$

Pyronaridine-artesunate is known to cause asymptomatic mildto-moderate transient increases in liver transaminases in some malaria patients. ${ }^{5,8}$ A limitation of the current study was that hepatic enzyme levels were not monitored. The standard protocol for therapeutic efficacy studies does not require laboratory monitoring, although there is provision for specific safety measures to be included if required. However, a recent extensive investigation showed no increase in the risk of hepatic transaminase elevations on repeated pyronaridine-artesunate dosing, and no clinical manifestations of hepatic injury associated with the biochemical observations. ${ }^{6,7}$ There were also no clinical indications of any hepatic adverse effect of pyronaridine-artesunate in the current study.

In conclusion, this study evaluated pyronaridine-artesunate efficacy against $P$. falciparum and $P$. vivax malaria in Myanmar. There were no cases of treatment failure for either parasite, and the therapy was well tolerated. Pyronaridine-artesunate could be added to the national malaria treatment guidelines in Myanmar and can be considered as an additional tool for programmatic decisions aimed at achieving malaria elimination for both $P$. falciparum and $P$. vivax.

Received March 11, 2020. Accepted for publication April 21, 2020.

Published online June 8, 2020.

Acknowledgments: We thank Naomi Richardson of Magenta Communications Ltd. who developed a first draft of this article from the statistical output and study reports, collated author contributions, and provided graphic services and was funded by Medicines for Malaria Venture. We also thank the Shin Poong Pharmaceutical Co., Seoul, Republic of Korea, for donating pyronaridine-artesunate. We thank the Pasteur Institute in Cambodia, Phnom Penh, Cambodia, for validating the molecular surveillance data.

Financial support: This work was supported by the Bill \& Melinda Gates Foundation and USAID-PMI through the WHO.

Disclaimer: The funding source was not involved in the design and conduct of the study, interpretation of the results, and development of this publication. B. T., D. M. B., and P. R. are staff members of the WHO. The authors alone are responsible for the views expressed in this publication, and they do not necessarily represent the decisions, policy, or views of the WHO.

Disclosures: I. B.-F. and S. D. are employees of Medicines for Malaria Venture.

Authors' addresses: Kay Thwe Han, Parasitology Research Division, Department of Medical Research, Yangon, Myanmar, E-mail:drkaythwehan@ yahoo.com. Khin Lin, Department of Medical Research (Pyin Oo Lwin Branch), Myanmar, E-mail: dr.khinlin.dir@gmail.com. Zay Yar Han, Department of Medical Research, Ministry of Health and Sports, Yangon, Myanmar, E-mail: zayyar.han@duke.edu. Moe Kyaw Myint, Parasitology Research Division, Department of Medical Research (Pyin Oo Lwin Branch), Myanmar, E-mail: dr.myintmoekyaw@gmail.com. Kyin Hla Aye, Department of Medical Research, Ministry of Health and Sports, Yangon, Myanmar, E-mail: kyinhlaaye@gmail.com. Aung Thi, National Malaria Control Programme, Department of Public Health, Ministry of Health and Sports, Myanmar, E-mails: aungthi08@gmail.com or 
aungthi@mohs.gov.mm. Badri Thapa, Malaria Control, World Health Organization, Yangon, Myanmar, E-mail: thapab@who.int. Maria Dorina Bustos, WHO Country Office for Thailand, Ministry of Public Health, Nonthaburi, Thailand, E-mail: bustosm@who.int. Isabelle BorghiniFuhrer, Product Development, Medicines for Malaria Venture, Geneva, Switzerland, E-mail: borghinii@mmv.org. Pascal Ringwald, Global Malaria Programme, World Health Organization, Geneva, Switzerland, E-mail: ringwaldp@who.int. Stephan Duparc, Medicines for Malaria Venture, Geneva, Switzerland, E-mail: duparcs@mmv.org.

This is an open-access article distributed under the terms of the Creative Commons Attribution (CC-BY) License, which permits unrestricted use, distribution, and reproduction in any medium, provided the original author and source are credited.

\section{REFERENCES}

1. Menard D et al., 2016. A worldwide map of Plasmodium falciparum K13-propeller polymorphisms. N Engl J Med 374: 2453-2464.

2. Straimer J et al., 2015. Drug resistance. K13-propeller mutations confer artemisinin resistance in Plasmodium falciparum clinical isolates. Science 347: 428-431.

3. Amato R et al., 2018. Origins of the current outbreak of multidrugresistant malaria in southeast Asia: a retrospective genetic study. Lancet Infect Dis 18: 337-345.

4. van der Pluijm RW et al., 2019. Determinants of dihydroartemisininpiperaquine treatment failure in Plasmodium falciparum malaria in Cambodia, Thailand, and Vietnam: a prospective clinical, pharmacological, and genetic study. Lancet Infect Dis 19: 952-961.

5. Pryce J, Hine P, 2019. Pyronaridine-artesunate for treating uncomplicated Plasmodium falciparum malaria. Cochrane Database Syst Rev 1: CD006404.

6. West African Network for Clinical Trials of Antimalarial Drugs, 2018. Pyronaridine-artesunate or dihydroartemisinin-piperaquine versus current first-line therapies for repeated treatment of uncomplicated malaria: a randomised, multicentre, open-label, longitudinal, controlled, phase 3b/4 trial. Lancet 391: 1378-1390.

7. Sagara I et al., 2016. Safety and efficacy of re-treatments with pyronaridine-artesunate in African patients with malaria: a substudy of the WANECAM randomised trial. Lancet Infect Dis 16: 189-198.

8. Duparc S, Borghini-Fuhrer I, Craft CJ, Arbe-Barnes S, Miller RM, Shin CS, Fleckenstein L, 2013. Safety and efficacy of pyronaridine-artesunate in uncomplicated acute malaria: an integrated analysis of individual patient data from six randomized clinical trials. Malar J 12: 70.

9. Kayentao K et al., 2012. Pyronaridine-artesunate granules versus artemether-lumefantrine crushed tablets in children with Plasmodium falciparum malaria: a randomized controlled trial. Malar J 11: 364

10. Rueangweerayut $R$ et al., 2012. Pyronaridine-artesunate versus mefloquine plus artesunate for malaria. $N$ Engl $\mathrm{J}$ Med 366: 1298-1309.

11. Tshefu AK et al., 2010. Efficacy and safety of a fixed-dose oral combination of pyronaridine-artesunate compared with artemether-lumefantrine in children and adults with uncomplicated Plasmodium falciparum malaria: a randomised non-inferiority trial. Lancet 375: 1457-1467.

12. Poravuth $Y$ et al., 2011. Pyronaridine-artesunate versus chloroquine in patients with acute Plasmodium vivax malaria: a randomized, double-blind, non-inferiority trial. PLoS One 6: e14501.

13. Leang Ret al., 2016. Efficacy and safety of pyronaridine-artesunate for treatment of uncomplicated Plasmodium falciparum malaria in western Cambodia. Antimicrob Agents Chemother 60: 3884-3890.

14. Leang R, Khim N, Chea H, Huy R, Mairet-Khedim M, Mey Bouth D, Dorina Bustos M, Ringwald P, Witkowski B, 2019. Efficacy and safety of pyronaridine-artesunate plus single-dose primaquine for the treatment of malaria in western Cambodia. Antimicrob Agents Chemother 63: e01273-19.

15. Leang R, Mairet-Khedim M, Chea H, Huy R, Khim N, Mey Bouth D, Dorina Bustos M, Ringwald P, Witkowski B, 2019. Efficacy and safety of pyronaridine-artesunate plus single-dose primaquine for treatment of uncomplicated Plasmodium falciparum malaria in eastern Cambodia. Antimicrob Agents Chemother63: e2242-18.
16. Bui Quang P et al., 2020. Pyronaridine-artesunate efficacy and safety in uncomplicated Plasmodium falciparum malaria in areas of artemisinin-resistant falciparum in Viet Nam (2017-2018). Clin Infect Dis 70: 2187-2195.

17. World Health Organization, 2019. World Malaria Report. Available at: https://www.who.int/publications-detail/world-malaria-report2019. Accessed February 8, 2020.

18. Chaumeau $V$ et al., 2018. Entomological determinants of malaria transmission in Kayin state, eastern Myanmar: a 24-month longitudinal study in four villages. Wellcome Open Res 3: 109.

19. Phommasone $\mathrm{K}$ et al., 2020. Mass drug administrations with dihydroartemisinin-piperaquine and single low dose primaquine to eliminate Plasmodium falciparum have only a transient impact on Plasmodium vivax: findings from randomised controlled trials. PLoS One 15: e0228190.

20. Guthmann JP, Pittet A, Lesage A, Imwong M, Lindegardh N, Min Lwin M, Zaw T, Annerberg A, de Radigues X, Nosten F, 2008. Plasmodium vivax resistance to chloroquine in Dawei, southern Myanmar. Trop Med Int Health 13: 91-98.

21. Marlar T, Myat Phone K, Aye Yu S, Khaing Khaing G, Ma S, Myint O, 1995. Development of resistance to chloroquine by Plasmodium vivax in Myanmar. Trans R Soc Trop Med Hyg 89: 307-308.

22. Xu $S$ et al., 2019. Efficacy of directly-observed chloroquineprimaquine treatment for uncomplicated acute Plasmodium vivax malaria in northeast Myanmar: a prospective open-label efficacy trial. Travel Med Infect Dis 101499.

23. Nyunt MH, Han JH, Wang B, Aye KM, Aye KH, Lee SK, Htut Y, Kyaw MP, Han KT, Han ET, 2017. Clinical and molecular surveillance of drug resistant vivax malaria in Myanmar (2009-2016). Malar J 16: 117.

24. World Health Organization, 2019. Template Protocol for Therapeutic Efficacy Studies. Available at: https://www.who.int/malaria/ areas/template-protocol-for-tet-en.docx?ua=1. Accessed February 1, 2020.

25. World Health Organization, 2000. Severe falciparum malaria. World Health Organization, Communicable Diseases Cluster. Trans $R$ Soc Trop Med Hyg 94 (Suppl 1): S1-S90.

26. World Health Organization, 2009. Methods for Surveillance of Antimalarial Drug Efficacy. Available at: https://www.who.int/ malaria/publications/atoz/9789241597531/en/. Accessed February $2,2020$.

27. World Health Organization, 2008. Methods and Techniques for Clinical Trials on AntimalariaOl Drug Efficacy: genotyping to Identify Parasite Populations. Available at: http://www.who.int/ malaria/publications/atoz/9789241596305/en/. Accessed February 26, 2019

28. Ariey $\mathrm{F}$ et al., 2014. A molecular marker of artemisinin-resistant Plasmodium falciparum malaria. Nature 505: 50-55.

29. Phompradit $P$, Chaijaroenkul $W$, Muhamad $P$, Na-Bangchang $K$, 2019. K13 propeller domain mutations and pfmdr1 amplification in isolates of Plasmodium falciparum collected from Thai-Myanmar border area in 2006-2010. Folia Parasitol (Praha) 66: 2019.006.

30. Zhao $Y$ et al., 2019. Genetic variations associated with drug resistance markers in asymptomatic Plasmodium falciparum infections in Myanmar. Genes (Basel) 10: E692.

31. Shetty AC et al.; Artemisinin Resistance Confirmation C, Containment, Artemisinin Resistance C, Elimination, Tracking Resistance to Artemisinin C, 2019. Genomic structure and diversity of Plasmodium falciparum in southeast Asia reveal recent parasite migration patterns. Nat Commun 10: 2665.

32. Tun KM et al., 2015. Spread of artemisinin-resistant Plasmodium falciparum in Myanmar: a cross-sectional survey of the K13 molecular marker. Lancet Infect Dis 15: 415-421.

33. Han KT et al., 2020. Artemether-lumefantrine and dihydroartemisininpiperaquine retain high efficacy for treatment of uncomplicated Plasmodium falciparum malaria in Myanmar. Am J Trop Med Hyg 102: 598-604.

34. Myint MK, Rasmussen C, Thi A, Bustos D, Ringwald P, Lin K, 2017. Therapeutic efficacy and artemisinin resistance in northern Myanmar: evidence from in vivo and molecular marker studies. Malar J 16: 143.

35. Geng J et al., 2019. Increasing trends of malaria in a border area of the greater mekong subregion. Malar J 18: 309. 